\title{
Sprachliche Physiognomie als linguistische Kategorie Möglichkeiten einer linguistischen Operationalisierung des Physiognomiebegriffs ${ }^{1}$
}

\section{Sprachliche Physiognomie bei Walter Benjamin}

Am Ende einer Rezension mit dem Titel Probleme der Sprachsoziologie steckt Walter Benjamin einen sprachtheoretischen Stoffkreis ab, der den der Sprache »innewohnenden Ausdruckscharakter « ${ }^{2}$ zum Zentrum hat. Er würdigt die "Sprachphysiognomik « ${ }^{3}$ als Überwindung der von ihm befehdeten "onomatopoetischen Theorie ${ }^{4}{ }^{4}$ die das mimetische Potential der Sprache in der phonologischen Imitation einzelner Momente verwirklicht sieht, und plädiert dafür, Ähnlichkeit in einem weniger unmittelbaren Sinne als Orientierungspunkt eines Verhaltens zu fassen, das einem opaken X Gestalt zu verleihen sucht. Darüber, was man sich unter einem solchen Verhalten vorzustellen hat, bietet der Text mit seinen eher aperçuartigen Ausführungen keinen näheren Aufschluss. Es wird jedoch zumindest die Richtung gewiesen, die Benjamins Überlegungen nehmen, wenn nämlich nach einem MallarméZitat die Bemerkung fällt, dass erst eine "Anschauung, die die Wurzeln des sprachlichen und des tänzerischen Ausdrucks in ein und demselben mimetischen Vermögen erblickt«, den Beginn einer validen sprachphysiognomischen Forschung markiere. ${ }^{5}$ Freilich mutet auch dieses Diktum noch enigmatisch an; es zu dechiffrieren, ist ohne Konsultation anderer Texte Benjamins kaum möglich. Darauf, welcher hier etwa in Betracht käme, gibt Benjamin einen Fingerzeig, wenn er in einem Brief an Werner Kraft über sein Referat Folgendes sagt: »[E]s ist von mir [...] so eingerichtet, daß es genau

1 Der Autor ist Hans-Georg von Arburg für vielfältige Hinweise und Verbesserungsvorschläge zu großem Dank verpflichtet.

2 Walter Benjamin: Probleme der Sprachsoziologie. Ein Sammelreferat, in: ders.: Gesammelte Schriften. Unter Mitwirkung von Theodor W. Adorno und Gershom Scholem hg. von Rolf Tiedemann und Hermann Schweppenhäuser, Bd. III, Frankfurt a.M. 1972, S. 452-480, hier S. 479.

3 Ebd., S. 478.

4 Ebd.

5 Ebd. 
an die Stelle führt, wo meine eigene Sprachtheorie, die ich auf Ibiza [...] in einer ganz kurzen programmatischen Notiz niedergelegt habe, einsetzt.« ${ }^{6}$ Die Herausgeber der Gesammelten Schriften identifizieren das genannte Dokument mit einer exposéartigen Schrift, welche in zwei Versionen vorliegt, die Lehre vom Ähnlichen bzw. Über das mimetische Vermögen überschrieben sind. Die Untersuchung wird sich im Weiteren auf die erste, umfänglichere dieser beiden 1933 entstandenen Fassungen stützen, die ins Mystische hinüberspielt, während das esoterische Moment in der zweiten abgemildert, nicht aber ausgeschaltet ist. ${ }^{7}$

Benjamin gewahrt in dem »Produzieren von Ähnlichkeiten« eine intuitive Reaktion auf "natürliche" oder "magische Korrespondenzen ${ }^{8}$ zwischen Sachverhalten und Geschehnissen, wie sie in vorgeschichtlicher Frühzeit, in der er den Ursprung des mimetischen Vermögens verortet, den »Mikro«ebenso wie den »Makrokosmos" organisiert haben sollen. ${ }^{9} \mathrm{Im}$ mimetischen Vermögen nimmt der apperzeptive Mechanismus des Menschen Fühlung mit dem prima vista abgezirkelten Reservat des Ontischen auf. Dieser Kontakt wird freilich primär von den Dingen her avisiert; eine Passage aus der Lehre vom Ähnlichen legt die Deutung nahe, dass von den Ähnlichkeiten, die der Seite des ontisch Gegebenen zuzurechnen sind, eine Aufforderung zu dessen Erkundung ergeht, welcher der Mensch entspricht, indem er nach jenen Ähnlichkeiten zu suchen beginnt: Sie »[...] erhalten die entscheidende Bedeutung erst im Licht der Überlegung, daß sie alle [...] Stimulantien und Erwecker jenes mimetischen Vermögens sind, welches im Menschen ihnen Antwort gibt «. ${ }^{10}$

Um diese Gedankenfigur eines mimetischen Vermögens stärker zu profilieren, referiert Benjamin auf die Astrologie, deren Gegenstand er prinzipiell "Nachahmbarkeit «11 zuschreibt, worunter er dem soeben Ausgeführten entsprechend eine »Anweisung « begreift, »eine vorhandene Ähnlichkeit zu handhaben «. ${ }^{12}$ Er bescheinigt den Sternbildern mithin latente Intentionalität

6 Walter Benjamin: Briefe, hg. und mit Anm. versehen von Gershom Scholem und Theodor W. Adorno, Bd. 2, Frankfurt a.M. 1966, S. 705.

7 Vgl. ders.: Gesammelte Schriften, Bd. II/3 (Anmerkungen der Herausgeber), Frankfurt a.M. 1977, S. 950.

8 Ders.: Lehre vom Ähnlichen/Über das mimetische Vermögen, in: ders.: Gesammelte Schriften, Bd. II/1, Frankfurt a.M. 1977, S. 204-213, hier S. 205 bzw. 206. Ebd., S. 205.

10 Ebd.

11 Ebd., S. 206.

12 Ebd. 
im Wortsinne, nämlich verstanden als Gerichtetheit auf ein exploratives Subjekt, das von ihnen zu »kultischen Veranstaltungen « wie dem Tanz ${ }^{13}$ bewogen wird. So gelangt Benjamin dazu, die »Nachahmbarkeit« des Gegenstandes »durch den Menschen« und das »mimetische Vermögen" durch die Partikel »bezw.« als Äquivalente miteinander zu verschalten, ${ }^{14}$ wenn nicht einander gleichzusetzen; das »Vermögen« deklariert er über den Relativsatz »das dieser hat " hierbei nochmals als Possessum des Menschen, so dass der Anschein einer gewissen Redundanz entsteht: Es ist, als gehe es Benjamin darum, möglichst klar herauszuarbeiten, dass die Mimesis grundsätzlich einer intentionalen Steuerung unterliegt. Die Ähnlichkeit nun, an die sich die Astrologie zu halten hat, ist die "zwischen einer Sternkonstellation und einem Menschen «15 und somit nicht ohne weiteres augenfällig. Dies mag den Anstoß dazu gegeben haben, dass Benjamin sie für ephemer erklärt, ${ }^{16}$ freilich ohne ihren Ähnlichkeitscharakter zu dementieren. Der Mangel an Selbstevidenz, der diese Ähnlichkeit unweigerlich depotenziert, rührt daher, dass der Mensch kein Organ mehr für sie hat. Ist die Ähnlichkeit zum strukturellen Substrat verkümmert, so ergibt sich daraus, dass der Schwund des mimetischen Vermögens auf die Homologie zurückgestrahlt und sich ihr als objektiver Index mitgeteilt hat: Die Ähnlichkeit, die nicht mehr als Ähnlichkeit erkannt wird, ist "unsinnlich«, und zwar so, als ob sie es von jeher gewesen wäre; denn sie nicht erfassen, heißt keinen Wink mehr von ihr empfangen, wie eine anschauliche Vorstellung sich aus ihr entwickeln lässt. Benjamin exemplifiziert die Idee einer "unsinnlichen Ähnlichkeit» an der Sprache, die er als deren "Kanon« oder paradigmatische Manifestationsform bezeichnet, und sucht auf diesem Wege einen weiten Mimesisbegriff zu lancieren, der seiner Forderung gemäß auf die Sprache als Ganze Anwendung findet. Dabei gerät eher noch als die mündliche Rede mit ihren »Lautzusammenstellungen", die nach Benjamin ihrem signifié homolog sind, die Schrift, genauer das »Verhältnis des Schriftbildes von Wörtern oder Lettern zu dem Bedeuteten bezw. dem Namengebenden ${ }^{\prime 17}$ in den Blick. Eine Betrachtung der Schrift wird dieser Anschauung zufolge darauf führen, dass die "Verspannung nicht zwischen dem Gesprochnen und Gemeinten sondern auch zwischen dem Geschriebnen und Gemeinten und gleichfalls zwischen dem

14 Ebd., S. 206.

15 Ebd., S. 207.

16 Vgl. ebd., S. 206.

17 Ebd., S. 208. 
Gesprochnen und Geschriebenen« aus der unsinnlichen Ähnlichkeit heraus motiviert ist und diese bezeugt. ${ }^{18}$ Als erstes Ergebnis ist hier zu vermerken, dass es sich bei den Gliedern, zwischen denen ein unterschwelliges Korrespondenzverhältnis vorwaltet, um Abstrakta von flächig-globaler Struktur handelt, die sich nicht in distinkte Einzelmomente wie Wörter und deren Referenzgrößen zergliedern lassen. Das erhellt aus den substantivierten Partizipien "Gesprochnem", "Geschriebnem « und "Gemeintem«, in denen sich eine sprachtheoretische Position abdrückt, die weniger die Zeichen und ihre Bedeutungen als den Zusammenhang für relevant erachtet, in den sie aufgehen, um in den Dienst einer Ausdrucksintention zu treten. Die Partizipien schälen aus den materiellen Zeichen und ihren immateriellen Korrelaten jeweils kollektive Einheiten heraus und designieren sie als Derivate von Handlungen des Sprechens, Schreibens und Meinens. Laut- und Schriftsprache werden primär als Prozessgestalt der Äußerung und nicht als statisches System gedacht, dessen Elemente einer vorgefertigten Verknüpfungslogik gemäß, deren Blaupause etwa die Grammatik abgibt, miteinander kombiniert sind. Die Ähnlichkeiten können bereits deshalb nicht anschaulicher Natur sein, weil zu ihrer Entdeckung eine perspektivische Voreinstellung nötig ist. Diese Voreinstellung lässt das, was da einander ähnlich sein soll, nur als Totale in den Gesichtskreis treten, welche das Detail - Laut bzw. Buchstabe, Wort, Satz usw. - überstrahlt. Sprache, so ergibt sich daraus, ist bei Benjamin von vornherein als Abstraktion von der sprachlichen Handlung gedacht. Damit ist auch der Differenzpunkt der auf das einzelne Sprachelement fixierten Onomatopoetik und der Sprachphysiognomik aufgedeckt, die Benjamin propagiert. Diese Sprachphysiognomik verrät den Einfluss des Psychologen Heinz Werner, dessen Monografie mit dem Titel Grundfragen der Sprachphysiognomik Benjamin in der eingangs erwähnten Rezension Probleme der Sprachsoziologie streift. Er spendet ihr dort Beifall, moniert jedoch in einer nachgelassenen Notiz, dass Werner die historische Dimension seines Sujets weitgehend ausgeblendet habe. ${ }^{19}$ Das Argument, dass man "die eigentümliche Dynamik, welche durch das Ganze zieht«, erspüren müsse, um das Besondere eines Gesichtes zu identifizieren, ${ }^{20}$ markiert die Linie, auf der die Überlegungen Benjamins sich bewegen. Sie münden in die Ansicht ein, dass die Ähnlichkeit in der Sprache sich erst enthülle, wenn man von dem in die Bewegtheit des Schriftbildes Eingeformten, d.h. von dessen nur

18 Ebd. - In der zweiten Fassung steht der Ausdruck »Verspannungen« im Plural (S. 212).

Heinz Werner: Grundfragen der Sprachphysiognomik, Leipzig 1932, S. 3. 
schattiertem Sinn, einen Begriff hat. Bei Werner heißt es, dass in der Schrift wie in der mündlichen Sprache eine "Sinndynamik « Gestalt annehme und »die optischen Linienzüge «21 als »sichtbar gewordene Artikulation, sichtbar gewordene Tongebärde «22 zu rezipieren seien, damit sie ihren vollen Gehalt preisgäben.

Diese Charakterisierung des physiognomischen Potentials der Schrift verbreitet Licht über eine Stelle in Benjamins Lehre vom Ähnlichen, die sich nicht ohne weiteres dem von den oben referierten Passagen gespannten Bogen unterordnet. Gleich nach der Exposition der "Verspannungen", aus denen die »unsinnliche Ähnlichkeit« hervorkeime und die in einer Übergangszone zwischen Materiellem und Immateriellem zu lokalisieren seien, wird - wenn auch unter Verwendung des Modalverbs »dürfen«, das obendrein im Konjunktiv steht - mit dem Konnex »Zwischen dem Geschriebnen und Gesprochnen« die mündliche Rede als semiotische Schaltung zwischen die Schrift und ihr außersprachliches Substrat geschoben. Auch dieser Schritt hat ein Pendant bei Werner, wie bereits dessen Räsonnement über die Schrift zu entnehmen ist, wo diese unter dem Stichwort "sichtbar gewordene Tongebärde" firmiert. Ihm zufolge gelangt die "sinnhafte Dynamik als Gesicht der Dinge zuerst in der tönenden und leiblich artikulierten Sprache «23 zur Evidenz und erschöpft die Leistung der Schrift sich darin, den "schon sprachgewordenen Ausdruck « ${ }^{24}$ nochmals aus einem anderen Medium herauszubilden, und zwar im Verlaufe eines Prozesses, der mit der Hervorbringung einer Plastik enggeführt wird: Werner apostrophiert jene Prägung der Schrift, die sie befähigt, die der Sprache vorgängige "sinnhafte Dynamik « zu konkretisieren, als Ergebnis eines "Knetens" und hebt vermittels des Kompositums "Wortmaterie« das stoffliche Moment der Schrift hervor, das sie zu dieser Tätigkeit disponiert. ${ }^{25}$ Dass die Schrift das ihr eingekörperte Immaterielle überspielen könnte, zieht Werner nicht in Erwägung. Er betont im Gegenteil, dass ein »Buchstabenzug" nicht als »optisch gegebenes Ornament» einzustufen sei, dessen Sinn in seinem Geformtsein läge. Als Stoff, in dem sich eine "im Artikulatorisch-Akustischen vorgeformte Dynamik " ausbilde, sei die Schrift »[...] nur von dem Organismus und System einer lautenden Sprache aus $\aleph^{26}$ adäquat zu erfassen. Aus dieser letzteren Bemerkung wird

21 Ebd., S. 70, Anm. 1.

22 Ebd., S. 72.

23 Ebd., S. 71.

24 Ebd., S. 72.

25 Ebd., S. 71.

26 Ebd., S. 72. 
nochmals ersichtlich, warum die physiognomische Praxis die Schrift als Ganzes behandeln soll, in dem das Einzelne verwischt ist: weil die in jener aufgespeicherte Energie zwar in Einzelmerkmalen konzentriert in Erscheinung treten mag, sich vollständig aber erst in der Bauform einer Sprache entbindet. Unter diesem Blickwinkel handelt es sich bei der Schrift um Materielles in einem reduzierten Maße, nämlich nur soweit Stofflichkeit vonnöten ist, um Abstraktes zu versinnlichen. Der Stoff scheint in das Letztere hineingezogen, so dass Stoffliches hier streng genommen bloß im Sinne einer vom Stoff abgespaltenen Qualität, als dessen Substrat Berücksichtigung erfährt. Dieser für die Sprachphysiognomik wesentlichen Problematik trägt Werner in den oben berührten Überlegungen zur Mimik Rechnung, indem er statuiert, dass der "Affektausdruck, den man gewöhnt ist, im Gesichte optisch aufzusuchen", nicht in den Bereich des "Sensoriellen« fällt, sondern »[̈̈]ber jede materielle Bestimmtheit hinaus existent« ist. ${ }^{27}$ Der »Ausdruck « partizipiert an einer »übergreifende[n] Totalität «, ${ }^{28}$ welcher (auch wenn Werner es nicht eigens erwähnt) Konkretheit nicht in dem Grade zukommt wie den Teilen, aus denen sie sich konstituiert. Denn das Wesentliche komplexer Zusammenhänge ist gerade dies, dass sie das Einzelne auf etwas Konzeptuelles ausrichten und jenem ihre Abstraktheit injizieren, indem sie es zu einer Funktionsgröße machen. Der "Ausdruck « ist als Element eines übergeordneten, geistigen Allgemeinen indes selbst nicht an eine "physische Substanz spezifisch gebunden ${ }^{29}$ und tendiert folglich nicht von sich aus zu einer prävalenten Erscheinungsform, so dass die Voraussetzungen, unter denen er sich realisiert, als unklar gelten müssen. ${ }^{30}$

Werners Reflexionen helfen bei der Erschließung der im Ganzen besehen doch recht enigmatisch anmutenden Lehre vom Ähnlichen, zumindest insoweit sie zu einer Klärung dessen beitragen, was es mit dem "Geschriebenen« bei Benjamin auf sich hat: Es ist dies ein Träger von Ähnlichkeit, dessen Medialität seine eigene materielle Erscheinung überstrahlt, so dass diese

27 Ebd., S. 7.

28 Ebd.

29 Ebd., S. 11.

30 Dieses Theorem gründet in der Vorstellung, dass sich in den einzelnen Wahrnehmungsarten eine vorgängige apperzeptive Totale ausdifferenziert; man könnte die Ähnlichkeiten, die Benjamin im Sinn hat, als deren Ausstrahlungen deuten und sich dabei auf ein Diktum Werners berufen, dem zufolge »Helligkeiten im Ton- und Vokalbereich jene urräumlichen dynamischen Spannungen besitzen, wie sie im Räumlich-Optischen bei Höhenunterschieden, im Akustischen bei >Tonigkeitsunterschieden auftreten« (Grundfragen der Sprachphysiognomik, S. 136f.). 
gleichsam hinter ihrer funktionalen Bestimmung verblasst. Anders als Werner jedoch, laut dem der "sprachliche Ausdruck « vor und unabhängig von "jeder sensoriellen Aufspaltung " existiert, ${ }^{31}$ räumt Benjamin wenigstens in der zweiten Version seines Textes der schriftlichen Sprache Vorrang vor der mündlichen ein - als biete jene bessere Bedingungen, um dem Ähnlichen zur Manifestation zu verhelfen, auch wenn dieses Ähnliche weder in den Graphemen noch in den Figuren, zu denen sie gruppiert sind, seine ursprüngliche Keimzelle hat. Benjamin weicht nämlich von Werner auch insofern ab, als er supponiert, dass das primäre Medium der unsinnlichen Ähnlichkeit, dasjenige, woraus sie als solche entstehe, sich seinerseits der Versinnlichung nach einer bestimmten Seite hin entziehe bzw. genau an jenem Indifferenzpunkt angesiedelt sei, der der Auffächerung des Ausdrucks in verschiedene Erscheinungsformen, also in Geschriebenes und Gesprochenes voraufliegt: Denn eben dies ist in der Bemerkung impliziert, dass das Mimetische "nur an etwas Fremdem, eben dem Semiotischen, Mitteilenden der Sprache« und damit, so schalten wir hier ein, an demjenigen, was an ihr unsinnlich ist, "als ihrem Fundus in Erscheinung tritt«. Dem lässt sich entnehmen, dass Benjamin die Referenzfunktion der Sprache, die hier in den Brennpunkt rückt, so wenig wie Saussure an die Gestalt oder Beschaffenheit einer sprachlichen Form zurückbindet, sondern sie vielmehr als ein Immaterielles und damit Anderes zu ihr hinzudenkt. Daraus, dass das Medium der Ähnlichkeit etwas gänzlich Abstraktes ist, das sich höchstens ansatzweise im Sprachmaterial auskristallisiert, erwachsen Konsequenzen für die Darstellung und das Dargestellte: Sie werden in Benjamins Diktum thematisch, dass in der Schrift weniger Bilder denn »Vexierbilder« Kontur gewönnen, die das »Unbewußte des Schreibers« in ihr »versteckt« habe ${ }^{32}$ und die allererst bloßgelegt werden müssten. Abgesehen von einem Hinweis auf die Graphologie fehlen freilich nähere Auskünfte dazu, wie das geschehen und was nach Beendigung des fälligen Procedere auf dem Bild zu erkennen sein soll. Immerhin aber wird klar, dass die unsinnliche Ähnlichkeit, auch wenn sie an das ideelle Substrat des Sprachsystems gebunden ist, ohne eine sinnliche Größe nicht zur Anschauung gelangen kann. Die mediale Seite der Sprache kommt mit anderen Worten nur dann zur Geltung, wenn sie eine Ausdrucksmaterie annektiert. Unbeschadet dessen ist die materielle Schicht gegenüber der medialen subsidiär, mit einem funktionalen Wert versehen und in ihrer Materialität getrübt. Diese Auffassung korrespondiert der Idee

32 Walter Benjamin: Lehre vom Ähnlichen, S. 209. 
Werners, wonach der Ausdruck in dem Sinne »hierarchisch geschichtet«33 ist, dass "über dem sinnlichen sich ein geistiger, über dem unmittelbaren ein mittelbarer Gehalt aufbaut«. ${ }^{34}$ Diese Schichtung zweier Größen, die nicht in einer dritten miteinander korreliert sind, findet bei Benjamin in dem Junktim zweier Präpositionalphrasen mit "aus " eine kongeniale sprachliche Realisierung. Der Primat des geistigen Moments vor dem sinnlichen wird dadurch angezeigt, dass der Relativsatz, der die beiden Phrasen enthält, als Teil einer prädikativen Konstruktion fungiert, die gerade das erstere Moment spezifizieren soll: "So ist der Sinnzusammenhang, der in den Lauten des Satzes steckt, der Fundus, aus dem erst blitzartig Ähnliches mit einem $\mathrm{Nu}$ aus einem Klang zum Vorschein kommen kann «. ${ }^{35}$ Dem wäre hinzuzufügen, dass das der Sprache immanente Potential, der Ausdruckssubstanz das Gemeinte zumindest temporär einzubilden, im mimetischen Vermögen der Sprecher sein Analogon hat. Nur bleibt Ersteres aufgrund der Übermacht des Kommunikativen als der sprachlichen Grundfunktion in dem Maße ungenutzt, wie Letzteres verschüttet ist. In der Instrumentalisierung der Sprache, die ihre mimetischen Impulse abschneidet und doch nur einem Wesenszug an ihr zu respondieren scheint, ist die Konditionierung der apperzeptiven Mechanismen in einer komplexen gesellschaftlichen Wirklichkeit mit Händen zu greifen. In beiden Prozessen zeichnet sich die gleiche historische Tendenzlinie ab.

Ist das Subjekt der Identifizierung von Ähnlichkeiten entwöhnt, behält es von diesen, wenn es sie doch einmal registriert, bloß einen diffusen Eindruck zurück. In einer entzauberten Wirklichkeit, die ihnen nicht länger einen höheren Sinn, geschweige denn Schicksalsmächtigkeit zubilligt, figurieren sie nur mehr als ephemere Signale. Zu deren Erkenntnis bedarf es einer hochgradig verfeinerten Auffassungsgabe: des Vermögens, die apperzeptiven Mechanismen auf Peripheres und Fluides einzustellen. ${ }^{36}$ Dass Ähnliches

33 Heinz Werner: Grundfragen der Sprachphysiognomik, S. 112.

34 Ebd., S. 117.

35 Walter Benjamin: Lehre vom Ähnlichen, S. 208f. [meine Hervorhebung, M.A.].

36 So ist nach der Lehre vom Ähnlichen das Erfassen unsinnlicher Korrespondenzen nicht ohne eine besondere "Schnelligkeit im Lesen oder Schreiben" denkbar, die als "Bemühen" oder "Gabe« angesprochen wird, »den Geist an jenem Zeitmaß teilnehmen zu lassen, in welchem Ähnlichkeiten [...] aus dem Fluß der Dinge hervorblitzen« (Walter Benjamin: Lehre vom Ähnlichen, S. 209). In einem anderen Passus, der sich einige Zeilen weiter oben findet, heißt es sinngemäß: Die "frühern Merkfähigkeiten für das Ähnliche" seien in die Sprache abgewandert, was bedeutet, dass das Vermögen zur Wahrnehmung des Ähnlichen von deren mimetischen Impulsen zu beziehen ist. Beim Lesen, bei welchem das signifié im Schriftbild entziffert wird, sind archaische Formen der Orientierung in der Welt wie die bereits genannte Sterndeuterei restituiert, für die der Glaube leitend sein soll, dass 
auch in der Sprache nur für Augenblicke die Wahrnehmungsschwelle erreicht, ratifiziert seine gesellschaftliche Marginalisierung, ist jedoch primär strukturell bedingt. Denn die materielle Substanz der Sprache ist, sobald sie ihre mimetische Seite hervorkehrt, in einem solchen Maße gegen ihre geistige Unterlage transparent, dass sie von dieser aufgezehrt scheint. Wo immer die mediale Qualität von Sprache zum Tragen kommt, ist ihre materielle Seite, wie oben erläutert, durch ihre konzeptuelle Funktionalisierung alteriert, von Geistigem überwölbt. So figuriert die Schrift bei Benjamin nicht in stofflicher Konkretheit, als Masse, der das Auszudrückende Form verleiht, sondern als »Archiv« und »Fundus «, ${ }^{37}$ mithin als Substrat der Materialisierung, das seines Teils von ihr freigehalten ist. In dieser Konstruktion eines ideellen Prinzips der Schrift, auf das alles Geschriebene zurückführbar sein soll, das gleichwohl nicht in diesem aufgeht, sich vielmehr als Abstraktes niemals völlig mit seinen empirischen Ausgestaltungen harmonisieren lässt, ist Derridas écriture-Begriff präfiguriert. In diesem Schriftbegriff, dessen Inhalt in eine empirische Normalform sowie ein geistiges Kondensat aufgebrochen ist, wendet die Prozedur der Terminologisierung sich offensiv gegen das factum brutum, dergestalt dass dieses von seinem reflexiven Insigne verdrängt wird. Bei Benjamin wiederum fällt an anderer Stelle, nämlich abermals in der Probleme der Sprachsoziologie überschriebenen Rezension, das Wort von den "physiognomischen Kräfte[n]« der Sprache,$^{38}$ das dem Geistigen an ihr eine

"Zwischen einer Sternkonstellation und einem Menschen« eine Ähnlichkeit bestehe, die sie aufzudecken hat. Benjamin begreift das Lesen in der Gegenwart als Endpunkt einer historischen Entwicklung, deren Anfänge in frühgeschichtlichen Bestrebungen liegen, aus natürlichen Phänomenen Aufschluss über die Zukunft zu gewinnen; dieser »okkulten Praxis" subsumiert er neben der Astrologie auch die Vogel- und Eingeweideschau, wohingegen Vorformen der modernen Schrift einem säkularen Lesen den Boden bereitet haben sollen. Die - gewiss gebotene - Problematisierung dieser Überlegungen müssen wir schuldig bleiben; der hier unternommene Exkurs soll stattdessen mit dem Hinweis schließen, dass sich die von Benjamin initiierte Mythisierung des Lesens unterschwellig in den Schriften Roland Barthes' fortsetzt und dort ebenso auf den Text wie auf die philologische Inventarisierung von dessen »Codes« ausweitet (vgl. Roland Barthes: S/Z, übers. von Jürgen Hoch, Frankfurt a.M. 1976, S. 18).

37 Wenn Benjamin bei der Behandlung der sprachlichen Mimesis Metaphern wie »abformen« ins Feld führt, so wirkt das wie ein Vorstoß, die Entstofflichung des mimetischen Mediums zu kompensieren: Wo es um die Spiegelung des Gemeinten in der Sprache geht, tritt, sei es auch nur verblümt-metaphorisch, der materielle Faktor auf den Plan; dies ist auch kaum anders denkbar, da mit der sprachlichen Aktualisierung eines signifié automatisch deren Form in den Brennpunkt der Betrachtung rückt.

38 Walter Benjamin: Probleme der Sprachsoziologie, S. 479. Diese Wendung ist innerhalb eines parataktischen Asyndetons mit dem Kompositum "Ausdruckscharakter" verklammert und somit als diesem synonym oder zumindest als ihm gleichsinnig ausgewiesen. 
energetische Fassung verleiht. Es wird supponiert, dass sich der Ausdruckswille, namentlich der künstlerische, von dem sich Wesentliches im Ornament mitteilt, als Triebfeder des Sprechens in der Sprache selbst konkretisiert. Benjamin trägt so der von Werner emphatisierten Dynamik im Bereich des Ausdruckshaften der Sprache Rechnung und insinuiert, dass in dieser eine bestimmte Schicht nach mimetischer Repräsentation des Bezeichneten strebt und in solcher Inklination latente Intentionalität oder zumindest immanente, nicht vom Subjekt induzierte Tendenzhaftigkeit augenfällig wird. Auch hier ist dem Theorem einer animierten Sprache als objektiver Wirkkraft, dem später ebenfalls Derrida seine kanonische Gestalt abgewonnen hat, die Bahn geebnet.

\section{Zur Sprach- und Zeichentheorie Roman Jakobsons}

Es sollen an dieser Stelle einige Überlegungen Roman Jakobsons eingeschaltet werden, der insofern als ein paradigmatischer Theoretiker der Sprachwissenschaft gelten darf, als manche konzeptuellen Universalien des Faches bei ihm eine mustergültige Ausprägung erfahren haben. Es ist daher zu erwarten, dass seine Arbeiten, sofern sie sich denn sinnvoll zu Benjamins Reflexionen ins Verhältnis setzen lassen, einen Weg zu deren linguistischer Operationalisierung weisen. Gleichwohl sei eingeräumt, dass man bei einem Vergleich Benjamins mit Jakobson Gefahr läuft, letztlich inkompatible theoretische Positionen gewaltsam einander anzugleichen und um vereinzelter Berührungspunkte willen, die erst auf ihre Belastbarkeit zu prüfen wären, die Unterschiede zwischen zwei Denkern zu nivellieren, die in denkbar heterogenen wissenschaftsgeschichtlichen Traditionen beheimatet sind. Solche Divergenzen sind an der Disposition wie an der sprachlichen Ausgestaltung der Schriften beider Autoren zu registrieren: Benjamins Texte sind hochspekulativ und dabei häufig montageartig gearbeitet; sie gründen in einem vielfach gestaffelten Unterbau und warten mit einer zwar konzentrierten, aber auch metaphorisch oszillierenden Begrifflichkeit auf, die den Terminus gegen die ihm subsumierten Merkmale transparent halten will. Jakobson hat dagegen primär materialphilologische Studien mit sehr technisch anmutenden Detailanalysen vorgelegt, in denen die Inventarisierung der Untersuchungsaspekte sich gegenüber dem sie umspannenden theoretischen Rahmen verselbständigt, und zwar mitunter so sehr, dass dieser Rahmen verblasst. Dem festgefügten terminologischen Kordon, der seine Aufsätze nach außen hin abriegelt, arbeiten aber auch bei Jakobson kaleidoskopische 
Darbietungsformen entgegen, wie sie Benjamin pflegt. Und so lassen sich bei allen Verschiedenheiten in der Anlage der Texte punktuelle Konvergenzen in der Form aufspüren, zu denen auch eine gewisse Ähnlichkeit im Gestus zählt: Beide Autoren gehen jeweils von einzelnen Ideen oder Befunden aus und gruppieren diese zu einem konfigurativen Tableau, das vielleicht stärker noch bei Jakobson als bei Benjamin etwas Mosaikartiges hat. Derartige Analogien, die keineswegs periphere Aspekte betreffen, legen die Vermutung nahe, dass es in den disparaten Denkgebäuden Schichten gibt, in denen sie unterschwellig miteinander kommunizieren; solche korrespondierenden Züge gilt es aufzudecken, wenn man die fraglichen Konzepte aufeinander beziehen möchte. Dies soll nachfolgend im Rekurs auf Jakobsons semiotische Reflexionen versucht werden. Damit geraten linguistische Problemstellungen in den Blick, auf die, obwohl sie sie kaum anschneiden, auch Benjamins sprachphysiognomische Räsonnements führen. Da Jakobson bei der Modellierung sprachwissenschaftlicher Größen an der materiellen Basis Maß nimmt, so dass es häufig den Anschein hat, als blieben seine theoretischen Kategorien den Daten verhaftet, darf man sich von seinen Arbeiten zudem einen Fingerzeig erhoffen, wie man dem sinnlichen Moment der Sprache, das gerade entschlossene Physiognomiker wie Benjamin und Werner größtenteils preisgaben, erneut zu seinem Recht verhelfen könnte.

Jakobsons semiotische Überlegungen sind Peirce verpflichtet, dessen Einteilung der Zeichen in Indizes, Ikone und Symbole er unverändert übernimmt. In einer Peirce gewidmeten Studie, die weitgehend referierenden Charakter hat, legt er den Akzent auf die Unterscheidung zwischen "signans" und "signatum « und insinuiert, dass die Dichotomie von materialer Substanz und "unmittelbarem Interpretanten" des Zeichens - der letztere Begriff stammt von Peirce ${ }^{39}$ - die paradigmatische Ausprägung von jener bildet. Diese schematische Gegenüberstellung konfligiert mit dem sprachphysiognomischen Axiom, wonach der sinnliche Ausdruck seinerseits mit Geistigem, also dem Peirce'schen Interpretanten, imprägniert ist, so dass die beiden Paradigmen, nach ihren Prämissen zu urteilen, schwer miteinander in Einklang zu bringen sind. Andere Partien von Jakobsons Aufsatz nötigen indes zu einer Korrektur dieser Folgerung, denn sie heben als zentral hervor, dass Peirce "zwischen den drei Grundklassen von Zeichen« lediglich Unterschiede »der relativen

39 Vgl. etwa Charles Sanders Peirce: Collected Papers, Bd. I-VI, hg. von Charles Hartshorne und Paul Weiss, Cambridge (Mass.) 1931-1935, CP 5.475. 
Hierarchie « 40 ansetzt, also keine undurchlässigen Scheidewände zwischen ihnen einzieht. Dementsprechend designieren die Zeichenbegriffe weniger an sich seiende Qualitäten denn funktionale Bestimmungen an solchen: Die Zeichen sind dadurch charakterisiert, dass sie potentiell alle diese Bestimmungen in sich tragen und dass ihre Verweisungsart von deren spezifischer Verteilung oder genauer davon, welche von ihnen vorherrscht, abhängig ist. Daraus darf man, auch wenn Jakobson selbst diesen Schritt nicht tut, ableiten, dass zwischen einem signifiant und seinem signifié mehrfach Verbindungslinien hin- und herlaufen, die an verschiedenen Ebenen des Ersteren angreifen und von denen eine, die auf eine besonders exponierte Stelle trifft, über die semiotische Valenz der Komplexion entscheidet. Daher können divergierende, ja gegensätzliche semiotische "Faktoren" wie "das Vorhandensein oder Fehlen von Ähnlichkeit oder Kontiguität zwischen signans und signatum" parallel nebeneinander existieren bzw. in dem Sinne zueinander ins Verhältnis treten, wie Jakobson meint, wenn er von "relative[r] Hierarchie« spricht. ${ }^{41}$ Für das Phänomen der Interferenz zweier Verweisungsarten ist nach Jakobson »ein zeigender Finger « qua »typischer Index « exemplarisch, den »in verschiedenen Kulturkreisen« differierende symbolische Informationen überlagern und in seiner Bedeutung modifizieren. So wird der Finger nicht überall wie in unseren Breitengraden als Aufforderung, dasjenige, worauf er deutet, näher ins Auge zu fassen, sondern wie etwa »in gewissen südafrikanischen Stämmen« als Verdikt verstanden. ${ }^{42}$ Umgekehrt ist in Symbole ein indexikalisches Element eingesprengt; ${ }^{43}$ reicht doch allein eine willkürliche Zuordnung von Signifikant und Signifikat, auch wenn sie sich verfestigt hat, nicht aus, um ein integrales Zeichen zu erzeugen: Die beiden Konstituenten verbinden sich nur unter der Bedingung zu einer Einheit, dass sie an einer bestimmten Stelle einander affin sind, dass der Signifikant etwas an sich hat, das darauf drängt, ihn einem Signifikat zuzuschlagen, und dass diese semiotische Anweisung sich in seiner materiellen Gestalt abdrückt. Man wird darin einen Versuch erblicken können, zwei zentrale sprachtheoretische Paradigmen, denen Platon im Kratylos einen prononcierten sprachlichen Ausdruck verlieh, um sie sogleich gegeneinander auszuspielen, die

40 Roman Jakobson: Suche nach dem Wesen der Sprache, in: ders.: Semiotik. Ausgewählte Texte 1919-1982, Frankfurt a.M. 1988, S. 77-98, hier S. 83.

41

Ebd.

Ebd., S. 84.

Siehe ebd. 
physei- und die thesei-Konzeption, miteinander zu versöhnen. In diesem Ziel gehen Peirce und Jakobson mit Benjamin und Werner einig. ${ }^{44}$

\section{Korrespondenzen zwischen Benjamin und Jakobson}

Im Weiteren sei nun herausgearbeitet, inwieweit die bisherigen Erläuterungen sich auf Benjamins Theoreme applizieren lassen. Das wohl wichtigste Resultat, auf das sie führen, lautet, dass das Zeichen sich nach Jakobson aus verschiedenen semiotischen Komponenten rekrutiert und die Zeichenarten lediglich als typisierende Hypostasen gelten dürfen. Wenn indes Symbole in diesem Sinne einen indexikalischen und Indices sowie Ikone einen symbolischen Faktor besitzen, so ist Bedeutung, freilich je in einem anderen Maße, ebenso an ein geistiges Moment - die willkürliche Verschränkung von signifiant und signifié - wie an das Zeichenmaterial gebunden. Diese Doppelstruktur hat das Zeichen, wie Jakobson es definiert, mit dem physiognomischen Ausdruck bei Benjamin gemein, der, wie oben dargetan, das Sinnliche seinerseits in etwas Immaterielles transponiert. Diese Parallele wird fasslicher, wenn man sich vergegenwärtigt, wie Jakobson sein Zeichenmodell für Textanalysen fruchtbar macht und welche Ausgestaltungen es in deren Verlauf erhält. Es tritt dann nämlich zutage, dass bei Jakobson ein ikonisches Element innerhalb eines Symbols in einer prinzipiell anders ausgerichteten oder gegensinnigen Zeichenkomplexion aufgeht und dieser zwar ein mimetisches Fluidum, nicht aber handfeste Bildlichkeit zuteilt noch sie in ihrer Verweisungsart umpolt. Wenn es schwer fällt, von einem solchen ikonischen Moment zu sagen, was es abbilden soll, so liegt das daran, dass die Vielschichtigkeit des Zeichens auf die Rechnung seines ontischen Korrelats zu setzen ist, es also die Vermitteltheit oder Abstraktheit des Sachhaltigen widerspiegelt. Es kommen denn auch häufig gar keine

44 Auch bei Theodor W. Adorno, der ebenfalls wichtige Überlegungen zu dem hier behandelten Stoffkreis geäußert hat, schimmert dieses Ziel bisweilen auf - so wenn er postuliert, philosophische Begriffe sollten eine Inklination zu den von ihnen avisierten Zusammenhängen besitzen oder sich um einen bestimmten Gehalt wie um eine semiotische Leerstelle herum gruppieren (vgl. Theodor W. Adorno: Negative Dialektik. Jargon der Eigentlichkeit, Frankfurt a.M. 1973 [Gesammelte Schriften, hg. von Rolf Tiedemann unter Mitwirkung von Gretel Adorno, Susan Buck-Morss und Klaus Schultz, Bd. 6], S. 167f.). Er synkopiert die Dichotomie von extrinsisch-arbiträrer und intrinsisch-mimetischer Bedeutungsstiftung mit der von Nominalismus und Realismus und weist an der hier verhandelten zeichentheoretischen Themenstellung eine epistemologische Tiefendimension auf, die Jakobson nirgends auch nur anzureißen scheint. 
Referenzobjekte in Betracht, die im Wortsinne abgebildet werden könnten, sondern nicht-konkrete, die bloß über umfunktionierte ikonische Momente gestalthaften Ausdruck erlangen. Dies sind nicht Gegenstände, sondern die Beziehungen, die sie zueinander unterhalten und deren diagrammatische Darstellung sich visueller Formen bedient, die von vornherein symbolisch determiniert sind und es zugleich der vom Symbol präformierten Aussageabsicht erlauben, passgenau an dem Gemeinten anzugreifen, indem sie das Repräsentationsformat strukturell auf es zuschneiden. Jakobson hat das Augenmerk vorzüglich auf sprachliche Formen der Strukturbildung gelenkt und so zumindest rudimentär ausgebildete diagrammatische Figuren im Sprachsystem freigelegt, die eine »isomorphe Anordnung von signans und signatum 45 auf der Ebene von Morphologie und Syntax erkennen lassen. Als Beispiel nennt er u.a. den »Ersatz tiefer Vokale oder Konsonanten durch helle, kompakter durch diffuse, andauernder durch nicht-andauernde, ungedeckte [sic] durch gedeckte (glottalisierte)«, der »in einigen amerikanischen Sprachen« eine Diminutiv- gegenüber der Normalform auszeichnet. Das Gegenstück dazu, das einen »augmentativen, intensiven Grad« repräsentiert, sind Phänomene, die sich »einem latenten synästhetischen Wert« verdanken, der phonologische Dichotomien zur Transponierung sinnlicher Impressionen und Empfindungen prädisponieren soll. ${ }^{46}$ Zur Illustrierung dieses Gedankens geht Jakobson im Rahmen eines Exkurses über Mallarmé auf dessen Unbehagen gegenüber den Lexemen jour und 'nuit ein, deren vokalische Qualität nicht mit ihren signifiés kongruiert und die Dichter wie eben Mallarmé, welche das phonetische Profil einzelner Ausdrücke in einen semiotischen Faktor umzumünzen wissen, zur Anbahnung einer Art semantischer Transkription nutzen. ${ }^{47}$

45 Roman Jakobson: Suche nach dem Wesen der Sprache, S. 87.

46 Ebd., S. 93.

47 Vgl. ebd., S. 94. Es sei hier bloß in Parenthese daran erinnert, dass der Name des französischen Symbolisten auch bei Benjamin fällt, und zwar gerade dort, wo dieser der Vision einer nicht-onomatopoetischen Sprachphysiognomik Raum gibt. Mallarmé, so resümiert Jakobson, kommt darauf zu sprechen, "daß das Wort ombre tatsächlich den Eindruck von Schatten vermittle, ténèbres jedoch (mit seinen hellen Vokalen) kein Gefühl der Dunkelheit suggeriere«; überdies habe er »die Zuweisung der Bedeutung >Tag« zu dem Wort jour und 'Nacht ‘u dem Wort nuit « als Täuschung empfunden, da jenes einen hellen und dieses einen dunklen Vokal enthalte. "Eine aufmerksame Lektüre der Tag- und Nachtbilder in der französischen Dichtung", so fährt Jakobson fort, "zeigt, wie nuit dunkler und jour heller wird, wenn nuit in der Umgebung eines Kontextes mit dunklen und tiefen Vokalen steht und wenn jour in einer Folge von hellen Phonemen aufgeht«. Französische Lyrik versieht »die >widersprüchlichen< Wörter mit geschickten Kontexten oder aber sie ersetzt 
Bekanntlich hat Jakobson den Begriff einer poetischen Funktion inauguriert, die in der Ausrichtung einer Mitteilung auf ihre sprachliche Form besteht. Das soeben Ausgeführte bietet eine Handhabe, diesem Konzept, dessen theoretische Unterlage Jakobson nur flüchtig umrissen hat, eine semiotische Drehung zu geben, etwa indem man statuiert, dass Dichtung, zumal die der Moderne, ihre Poetizität aus einem Ausdruckspotential gewinnt, das in vermeintlich nicht-signifikativen Größen des Sprachsystems angelegt und durch eine Verteilung der Lexeme, die sich an deren formalen Eigenschaften orientiert, zur Entfaltung gebracht ist. Das Konzept bewährt sich so als Ferment der poetischen Imagination, das sich in deren Formen und Inhalten als struktureller Einheitspunkt auskristallisiert. Als Potential aber muss es erst aktualisiert werden; das nicht-signifikative Material ist ausdrucksfähig und nicht selbst schon Ausdruck: Es wird zu einem solchen nur dann, wenn Dichtung es als Medium der Aussage auf selbige bezieht oder gegen sie in Stellung bringt, damit sich seine semantische Kraft entbindet. Jakobson konzediert der Konfigurierung der »Phoneme und ihrer Komponenten« eine »evokative Macht«, die zwar »im alltäglichen Sprachverhalten« spürbar, prinzipiell aber - und damit rührt er an den neuralgischen Punkt - „verborgen « sei. ${ }^{48}$ Dazu passt, dass Jakobson den "synästhetischen Wert« phonologischer Kontraste, wie oben sichtbar wurde, als "latenten", nicht ungebrochen vorhandenen deklariert und diese Bestimmung später nochmals dahingehend paraphrasiert, dass »das Ineinanderspielen von Laut und Bedeutung auf einer rein lexikalischen Ebene« lediglich eine »virtuelle« Erscheinung, hingegen »die intrinsische[,] diagrammatische Beziehung zwischen signans und signatum » »in Syntax und Morphologie«, mithin in Fällen wie dem der Steigerung oder Pluralbildung, "offensichtlich und obligatorisch" sei. ${ }^{49}$ An anderer Stelle kreist er den Hybridcharakter der mimetischen Kapazität der Formelemente ein, indem er mit Blick auf die handgreifliche Homologie im Bau von father, mother und brother davon spricht, dass sie bloß "als eine Art phonologische Anspielung « auf deren »semantische Verwandtschaft« »empfunden« werde..$^{50}$

das Bild des Tageslichtes und der Dunkelheit der Nacht durch die Gegenüberstellung eines schweren, drückenden Tages und einer luftigen Nacht, denn diese Gegenüberstellung wird durch eine weitere synästhetische Konnotation gestützt, die die tiefe Tonalität dunkler Phoneme mit Schwerem und entsprechend die hohe Tonalität heller Phoneme mit Leichtem assoziiert« (ebd.).

48 Ebd.

49 Ebd., S. 92.

50 Ebd., S. 91. 
Aus Äußerungen wie diesen erhellt, dass Jakobson mit Benjamin nicht bloß in der Idee einer Abbildungsfunktion der Sprache, sondern auch in dem Bestreben, diese Funktion zu depotenzieren, übereinstimmt: Bei beiden figuriert das Mimetische an der Sprache als ein zwar residuales, aber irreduzibles Moment, das freilich untrennbar an die sie überlagernde symbolische Kardinalfunktion gekettet ist. Das Mimetische hat die konventionelle Bedeutung als prototypische, allen zugängliche Repräsentation desjenigen Inhaltes nötig, dem die sprachliche Form sich angleichen soll: So erreichen lexikalische Konfigurationen mit dem französischen nuit als Zentrum, die die Atmosphäre einer »luftigen Nacht« erzeugen, ihre Wirkung nur deshalb, weil ihr semantischer Raum auf die konventionalisierte Extension des Lexems eingenordet ist. Dass es sich bei derartigen Formen sprachlicher Mimesis nicht oder nicht primär um onomatopoetische Erscheinungen handelt, liegt auf der Hand und ist als zweite, durchaus gewichtige Parallele zwischen Benjamin und Jakobson zu verbuchen. Indem Letzterer bei der Exegese die sprachlichen Einheiten unter- und oberhalb der lexikalischen Ebene berücksichtigt, liefert er Beispiele für Sprachgestalten, die das Gemeinte nicht, wie bei onomatopoetischen Versuchen üblich, durch plane Simulation auf der phonologischen Ebene, sondern als großflächigen Strukturzusammenhang und somit genau in dem Modus präsent halten, den Benjamin mittels des substantivierten Partizips "Geschrieb[e]nes" designiert. Signifikante Komplexe, wie sie Benjamin vorgeschwebt haben dürften, nimmt Jakobson vor allem in seinen Gedichtanalysen in Augenschein, die er auf die Prämisse gründet, dass sich in Konfigurationen grammatischer Bauformen deren latente Semantizität aktualisiert und dass man an diese Größen im Sinne der oben dargebotenen semiotischen Spekulationen die Kategorien von Index und Ikon anzulegen hat. Jakobsons Untersuchungen erbringen den Nachweis, dass das durch Kontiguität gestiftete Junktim von signifiant und signifié mitnichten selbstevident ist, sondern im hermeneutischen Rekurs auf das sprachliche Material nachvollzogen werden muss: Gelingt es, das Gemeinte an den Zeichen abzulesen und diese wiederum auf jenes zurückzubiegen, so kann man aus den gewonnenen Interferenzen zwischen Sprachlichem und Außersprachlichem den semiotischen Konnex selbst herausprozessieren. Jakobson sucht das durch rigide Zergliederung der materiellen Oberfläche der Referenztexte zu bewerkstelligen, wobei er auch vor einer ziemlich weitgehenden Umschichtung einzelner Konstituenten nicht zurückscheut: Diese Operationen dienen nicht allein analytischen Zwecken, sondern auch einer halb synthetischen Rematerialisierung jener latenten Gestaltqualität sprachlicher Gebilde, die Benjamin im Sinn hat, wenn er Sätzen eine Art von Ausdrucksfähigkeit 
attestiert, die sich in "Lautzusammenstellungen « bekundet, jedoch in einer Strukturidee unterhalb der sprachlichen Decke wurzelt, welche den Formationen ihren je spezifischen Gefügecharakter einverleibt. Da Jakobson das Lexem nicht mehr ausschließlich als Träger eines Bedeutungswertes, sondern als figuratives Element handhabt, stellt es bei ihm weniger eine untrennbare Einheit denn ein Ensemble von funktionalen Komponenten vor, die sich fortwährend neu gruppieren, so dass der von ihnen gebildete Verbund ein metamorphes Gepräge hat. Diese Konsequenz wird zum einen von der strukturalistischen Ausrichtung seiner Untersuchung präjudiziert und verlängert zum anderen die Linie der semiotischen Grundbestimmungen, die Jakobson von Peirce übernimmt. Dass der sprachliche Träger des Ausdrucks seine Materialität einbüßt, ist unter den inhaltlichen Zügen, die Jakobsons Überlegungen mit denen Benjamins verbinden, gewiss nicht der unwichtigste, denn von der Entstofflichung des lexikalischen Zeichens her fällt auf das enigmatische Attribut »unsinnlich«, welches das Spezifikum von Benjamins Ähnlichkeitskonzept bezeichnet, ein Licht. Ebenso wird deutlich, wie die Aussage, dass in der Schrift »Vexierbilder« versteckt seien, zu verstehen ist, wenn man sich vor Augen hält, wie persistent Jakobson die Decke eines Textes aufsprengt und den untergründigen Korrespondenzen zwischen seinen Einheiten nachforscht, um die mimetische Wirkungskraft der sprachlichen Form zu demonstrieren. Bei demjenigen, was ihm zufolge in die Lautkonfigurationen auf der Mikro- und Makroebene hineingespiegelt ist, handelt es sich um vielschichtige mentale Konstruktionen, in denen die empirische Wirklichkeit hinter den Resultaten ihrer konzeptuellen Verarbeitung fast völlig verblasst ist: In die Sprache sind, um die Brücke zu Benjamin zu schlagen, Bilder eingelegt, deren Gegenstand schlechterdings nicht auszumitteln ist und an die Erkennbarkeit des Dargestellten als Maßstab heranzutragen von vornherein verfehlt wäre. Ihre Abstraktheit ist im Falle Jakobsons jedoch auch Resultat eines Verfahrens, das systematisch von der sprachlichen Oberfläche der Texte abstrahiert und das Geistig-Semiotische, das Benjamin für das primäre Medium von Ähnlichkeit hält, gleichsam in seinen ersten Materialisierungsstufen aufzufinden sucht.

Unter diesem Gesichtspunkt kann man als Fazit dieser Studie festhalten, dass zum einen Jakobson ein Begriffsinventar zu einer linguistischen Kontextualisierung der nachgerade atemberaubend schwierigen, ja spekulativ-esoterischen Reflexionsfiguren Benjamins bereitstellt und dass zum anderen diese Letzteren eine sprachtheoretische Formulierung der allgemeinsten Prinzipien von Jakobsons analytischer Praxis ermöglichen. Gerade einer der einflussreichsten Theoretiker der Sprachwissenschaft bringt wesentliche Facetten des 
Benjamin'schen Physiognomiebegriffs in Ansatz, ohne ihn selbst zu denken; seine Interpretationen führen demgegenüber vor, wie sich das unter diesem Begriff Gedachte für die konkrete Spracharbeit fruchtbar machen lässt, und bringen so seine Relevanz für die linguistische Textexegese zur Evidenz. Dass die Sprachwissenschaft sich von einer Auseinandersetzung mit der Lehre vom Ähnlichen entscheidende Impulse erhoffen darf, liegt auf der Hand, wenn man bedenkt, dass mit der Frage nach dem Verhältnis von stofflicher Form und immaterieller Funktion, wie Benjamin sie aufwirft, ein Kernmoment innerhalb der Theoriebildung des Faches berührt ist. 